\title{
THE TETHERED GOAT - A CALCULUS APPROACH
}

\author{
Michael Brennan
}

The problem of the goat and the field was relayed to me by a friend who had been asked by a quantity surveyor to solve this seemingly simple problem of determining the rope length for a grazing goat so as the goat could graze half of a given field. The problem that I was given involved a circular field and at first seems to involve little more than doing some algebra on a standard area formula.

Some of my colleagues have heard a similar problem concerning grazing goats, except with regards to rectangular, square and even triangular fields. A reference to the triangular field dates back to 1917 and appears in [1]. The problem with the circular field is well known and recently appeared as a problem in the Mathematical Spectrum, [2, p.23]. In it, Andrew Lobb used a mix of geometry and trigonometry to solve the problem. Intuitively, even though these problems are clear, the mathematics is more involved than one would initially expect. Ultimately the problem reduces down to solving a transcendental equation which is best tackled by a Computer Algebra System like Maple or Mathematica. In our solution below, we use integral calculus to obtain an expression for the grazing area of the goat. Then by forming the appropriate equation, the problem is next to find the root of this equation.

\section{Setup}

Given a circular field of grass of diameter $100 \mathrm{~m}$. A farmer ties a goat to a pole on the circumference. When the rope is taut, the goat path sweeps out an arc of a circle of radius $r$. The problem is to determine the value of $r$ so that the area of the ground accessible to the goat is half the area of the circular field. First we need to 
find an expression in $r$ that gives the area of grass accessible to the goat. This area is the region enclosed by two circles, one of radius $r$ and the other of radius 50 .

The equation of the goat's circle (assuming that the rope pole is at $(0,0)$ and we measure the $x$-coordinate along the radial line to the centre of the circular field and the $y$-coordinate perpendicular to this line) is given by $x^{2}+y^{2}=r^{2}$, whereas the equation of the fixed field will be $(x-50)^{2}+y^{2}=50^{2}$. Using some elementary algebra we can find the intersection of these curves. The $x$-coordinate is all that is needed and this is found to be at $x=\frac{r^{2}}{100}$. The accessible area available to the goat can be found using integration. First we need to integrate over the region of the field under the curve defining the field from the pole, i.e. from $x=0$ to $x=\frac{r^{2}}{100}$. Then we integrate over the region under the curve that defines the goat's path from $x=\frac{r^{2}}{100}$ to $x=r$, which is the length of the rope. This area is the given by the following sum of two integrals

$$
\int_{0}^{\frac{r^{2}}{100}} \sqrt{50^{2}-(x-50)^{2}} d x+\int_{\frac{r^{2}}{100}}^{r} \sqrt{r^{2}-x^{2}} d x
$$

\section{Calculation}

Using the Computer Algebra System Maple V R4 [3], we can evaluate this integral sum to give an expression for the area. Note that due to symmetry the sum of the two integrals above needs to be multiplied by two to obtain an expression that represents the area that is accessible to the goat.

For Maple to evaluate the definite integrals correctly, we need first to make an assumption on the range of the variable $r$ (to avoid the incorrect introduction of complex numbers).

$>$ assume $(r<100)$

$>\mathrm{A}:=2 *\left(\operatorname{int}\left(\operatorname{sqrt}\left(50^{\wedge} 2-(\mathrm{x}-50) \wedge 2, \mathrm{x}=0 \ldots \mathrm{r}^{\wedge} 2 / 100\right)\right.\right.$

$\left.+\operatorname{int}\left(\operatorname{sqrt}\left(r^{\wedge} 2-x^{\wedge} 2\right), x=r^{\wedge} 2 / 100 \ldots r\right)\right)$; 


$$
\begin{aligned}
A:= & -\frac{1}{2} \sqrt{-r^{\sim 4}+10000 r^{\sim 2}}+2500 \arcsin \left(\frac{r^{\sim 2}}{5000}-1\right)+1250 \pi \\
& +\frac{1}{2} r^{\sim 3} \sqrt{\frac{1}{r^{2}}} \pi-r^{\sim 2} \arcsin \left(\frac{1}{100} \sqrt{\frac{1}{r^{2}}} r^{\sim 2}\right) .
\end{aligned}
$$

The tilde $\left(^{\sim}\right)$ on $r$ indicates that $r$ carries an assumption. Now we set this equal to one half of the area of field, $a=\frac{\pi 50^{2}}{2}$. Define a new function $B$ to be the difference between $A$ and $a$.

$>\mathrm{B}:=\mathrm{A}-50^{\wedge} 2 * \mathrm{Pi} / 2$

$$
\begin{aligned}
B:= & -\frac{1}{2} \sqrt{-r^{\sim 4}+10000 r^{\sim 2}}+2500 \arcsin \left(\frac{r^{2}}{5000}-1\right) \\
& +\frac{1}{2} r^{\sim 3} \sqrt{\frac{1}{r^{\sim 2}}} \pi-r^{\sim 2} \arcsin \left(\frac{1}{100} \sqrt{\frac{1}{r^{\sim 2}}} r^{22}\right) .
\end{aligned}
$$

\section{Answer}

All that is left to do is to find where $B$ has a root and this value will be the required length of rope. Intuitively we would expect this value to be greater than $50 \mathrm{~m}$. To find this value, we can use the Maple solve command. To obtain a floating point approximation, the numerical rootfinder fsolve will approximate the root.

$$
\begin{aligned}
& \begin{array}{l}
\text { solve }(\mathrm{B}=0, \mathrm{r}) \text {; } \\
100 \sin \left(\operatorname { R o o t O f } \left(4 \_Z \sin (-Z)^{2}-\arcsin \left(-1+2 \sin (-Z)^{2}\right)\right.\right. \\
\left.\left.\quad+2 \sqrt{-\sin (-Z)^{4}+\sin (-Z)^{2}}-2 \sin (-Z)^{2} \pi\right)\right) \\
\text { > f solve }(\mathrm{B}=0, \mathrm{r}) ; \\
\quad 57.93642365
\end{array}
\end{aligned}
$$

We conclude that the length of the rope is $\mathbf{r}=\mathbf{5 7 . 9 3 6 4 m}$, correct to four decimal places. A good homework exercise would 
be to solve the problem where the field is of a more conventional shape like a rectangle or a square. Note that for these problems the rope pole is located at one of the corners of the field.

\section{Acknowledgement}

The author would like to acknowledge the valuable assistance of Dr Tom Carroll, resulting in the necessary introduction of the assume command in the work above.

\section{References}

[1] H. E. Dudeney, Amusements in Mathematics. Dover, 1958.

[2] Andrew Lobb, Problem 29.7, Mathematical Spectrum 30 1997/8.

[3] K. M. Heal, M. L. Hansen, K. M. Rikard, Maple V, Learning Guide. Springer-Verlag, 1996.

Michael Brennan

Department of Mathematics

University College, Cork. 